\title{
Gracq et Buzzati: état de la question
}

\section{Norbert Germanaz}

\section{OpenEdition}

\section{Journals}

Édition électronique

URL : http://journals.openedition.org/studifrancesi/5836

DOI : 10.4000/studifrancesi.5836

ISSN : 2427-5856

\section{Éditeur}

Rosenberg \& Sellier

\section{Édition imprimée}

Date de publication : 1 mai 2011

Pagination : 128-137

ISSN : 0039-2944

\section{Référence électronique}

Norbert Germanaz, «Gracq et Buzzati: état de la question », Studi Francesi [En ligne], 163 (LV | I) | 2011, mis en ligne le 30 novembre 2015, consulté le 07 janvier 2021. URL : http://journals.openedition.org/ studifrancesi/5836 ; DOI : https://doi.org/10.4000/studifrancesi.5836

\section{(c) (i) (2) $\Theta$}

Studi Francesi è distribuita con Licenza Creative Commons Attribuzione - Non commerciale - Non opere derivate 4.0 Internazionale. 


\section{Gracq et Buzzati: état de la question}

Des années 1960 à ces dernières années 2000, les références à la proximité des deux romans Il Deserto dei Tartari et Le Rivage des Syrtes sont des «signaux intermittents» certes mais qui indiquent dans la durée l'association Gracq-Buzzati: côte à côte ou face à face, à travers communications, analyses comparatives, essais, allusions, opinions dispersées à travers revues et magazines. Nous ne rendrons compte que des principales contributions dont nous avons pu prendre connaissance, parfois anciennes, mais faisant autorité pour la critique comparative ${ }^{1}$. Nous suivrons, dans la mesure du possible, l'évolution de celle-ci à travers les ans sans exclure quelques entorses à la chronologie lorsque cela est nécessaire.

L'association Gracq-Buzzati se présente d'emblée comme un double mouvement de Gracq à Buzzati et de Buzzati à Gracq - des «impulsions de recherches» en quelque sorte. Ainsi l'auteur d'une communication au Colloque d'Angers en 1981 nous dit que ses «recherches dans le domaine de l'imaginaire lui ont fait comprendre la fascination exercée par Le Rivage des Syrtes dont je n'ai par la suite trouvé quelque équivalent que dans Le Désert des Tartares de Dino Buzzati» ${ }^{2}$ et garde de sa première lecture du Rivage des Syrtes une impression comme d'une tonalité dominante: celle de «l'attente enlisée et haletante à la fois», «ce qui explique d'ailleurs que Dino Buzzati me fasse irrésistiblement penser à Julien Gracq»².

\section{Les influences}

La question des influences s'est posée dès la parution et la diffusion en France du Rivage des Syrtes en 1951, par ailleurs couronné par le prix Goncourt en 1951 et la parution, bien plus tôt en Italie, de Il Deserto dei Tartari, en 1940, et sa traduction française datant de 1949 (chez Laffont). C'est donc en 1962 que Monique de Taeye-Henen ${ }^{3}$ suppose que la lecture de Il Deserto dei Tartari aurait influencé Gracq. «Le seul défaut de Gracq fut d'être le second» dit-elle. Et c'est en 1969 que Buzzati répond à une lettre adressée par Jean-Louis Leutrat. Il précise qu'il n'a lu de Gracq que Le Rivage des Syrtes; il note certaines analogies entre la situation des Syrtes et celle du Désert des Tartares; «dans les deux livres il y a une attente pratiquement sans fin; au-delà des frontières, sable ou mer, il n'y a pas de différence, vit un peuple très lointain et mystérieux, duquel peuvent venir des dangers et la mort», et il ajoute entre parenthèses «(est-ce que je ne commets pas là une erreur?). Toutefois l'atmosphère

(1) Nous n'avons pas pu accéder à la totalité de la critique sur le sujet, en particulier aux contributions italiennes et nous prions les lecteurs francophones et italiens de bien vouloir nous excuser.

(2) Simone Vierne, Actes du Colloque d'Angers (mai 1981), Julien Gracq, le mythe du Graal et la quête du sacré, Presses Universitaires d'Angers, 1981.

(3) Monique de Taeye-Henen, De l'invention romanesque à Pirandello, Paris, Didier, 1962. 
me semble complètement différente, soit dans ses caractéristiques pour ainsi dire physiques, soit dans le sentiment qu'on se propose de susciter» ${ }^{4}$.

On voit donc que les deux œuvres ont été assez vite rapprochées mais Gracq proteste en quelque sorte de son innocence et déclare ne pas avoir lu à ce moment-là Le Désert des Tartares: «La question du Désert des Tartares m’a été très souvent posée mais le livre n'a été traduit en français que quelques mois avant la parution du Rivage des Syrtes et je ne le connaissais pas quand j'ai écrit mon livre. Je suis en revanche redevable de quelque chose à Pouchkine pour le début de La Fille du Capitaine»s.

Par ailleurs on apprend qu'à la parution de la traduction française du Désert des Tartares en France en 1949 Le Rivage des Syrtes était alors pratiquement achevé à l'exclusion d'une soixantaine de pages. «La question des influences», qui a beaucoup agité la critique «est ainsi réglée par les faits» ${ }^{6}$. D'autre part on sait que Gracq a été très intéressé par l'œuvre de Junger à laquelle il a consacré deux articles ${ }^{7}$.

Quant à l'influence de Pouchkine, elle ne concerne pas seulement Gracq mais aussi Buzzati: telle est l'opinion d'Yves Frontenac. «Pour garder le lecteur en alerte, les trois romanciers (Pouchkine, Buzzati, Gracq) ont recours à une mise en haleine qui repose sur un procédé allusif et répétitif d'une efficacité absolue; les observateurs, bien avant l'assaut, détectent dans la steppe des feux qui deviennent chez Buzzati des lumières et chez Gracq des bruits. De même avant l'apparition des colonnes armées: apparitions brèves, autour de la forteresse de «cavaliers inconnus» (Pouchkine), de «patrouilles de reconnaissance» (Buzzati), de «nomades» (Gracq). Ce monde de l'attente cantonné dans la forteresse est à mi-chemin entre le solide et le fluide; d'une part un monde figé, rigide, minéral; de l'autre un monde en débâcle; tout fuit, tout reste indécis, sans contour précis» ${ }^{8}$.

Rappelons aussi une influence française reconnue de Buzzati: celle de Blaise Pascal. Michel Suffran rapporte dans son essai ces propos de Buzzati «Pascal est l'unique Français que je connaisse bien vraiment, je suis fasciné par son talent... Et il a une caractéristique, ce qu'il écrit a la limpidité de l'eau»".

Revenant à la revue L'Herne citée dans notre introduction et consacrée à Julien Gracq, d'où nous avons extrait la lettre de Buzzati, nous rendrons compte succinctement de l'article de Philippe Berthier «Gracq et Buzzati, Poètes de l'événement» (page 90).

Il s'agit d'une recherche du sens global des deux romans Rivage des Syrtes et Désert des Tartares, centrée sur la psychologie et le comportement des deux principaux protagonistes: Aldo et Drogo. Ce qui rapproche et ce qui sépare les deux œuvres est développé pour ainsi dire en contrepoint: «prélude à l'unisson en effet et de tonalité majeure: une joie indifférenciée qui éclatera plus tard en harmonies de plus en plus étrangères»: «Une prise de commandement» (Rivage des Syrtes); «première affectation» (Désert des Tartares), «L'atmosphère est lestée d'une conscience vaguement lourde de futur». «Mais Aldo par sa dérision volontaire, Giovanni (Drogo) par la densité de l'espérance dont il entoure un sort qui lui est imposé, se rangent d'emblée dans la race des âmes aux aguets de l'imminence» ${ }^{10}$.

(4) «L'Herne», Julien Gracq, 1972, Paris, Le Livre de Poche, 1987, page 103, Lettre adressée à $\mathrm{Ph}$. Berthier.

(5) Jean CARrière, Julien Gracq ou Les Reflets du Rivage, Gordes, Les Éditions du Relié, 2002, p. 161, entretien avec Gracq. Julien Gracq qui êtesvous? Entretiens avec Jean Carrière, Lyon, La Manufacture,1986, p. 117.

(6) Julien Grace, Euvres complètes, éd. Bernhilde BoIE, Tome I, Paris, Gallimard (La Pléiade),
1989, Notice p. 1327.

(7) J. GracQ, «Symbolique d'Ernst Junger» in Préférences, Paris, Corti, 1961.

(8) Yves Frontenac, Buzzati /Gracq: La séduction de Pouchkine, «Le Magazine Littéraire», octobre 1995 , p. 48.

(9) Michel Suffran, Dino Buzzati: le laboratoire secret, Tournai, La Renaissance du livre, 1998, Collection «Signatures».

(10) «L'Herne», cit., pages 90 à 104. 
Le cadre dans lequel se déroulent les deux récits «sable et eau» est «bien plus qu'un décor. C'est avant tout un espace intérieur, comme le désert pasolinien. C'est le lieu de la quête». Et Philippe Berthier souligne les différences dans les paysages: dans Le Désert des Tartares «roches déchiquetées, inhumaines, hostiles» et un fort «dans une sorte de gloire solaire insoutenable» ${ }^{11}$; dans Le Rivages des Syrtes, au contraire, Aldo est jeté dans le vertige de l'horizontalité «néanmoins fécondée par une sorte d'inhibition qui promet des efflorescences» ${ }^{12}$.

Mais l'auteur, dans le même mouvement, pointe aussi les éléments communs: «pourtant sur la mer des Syrtes comme sur le désert des Tartares pèse un même signe visible de l'attente: la brume». Toutefois si de l'Amirauté on voit de la brume, de l'autre côté de la brume, on voit «menace et merveille: le volcan - menaçant aussi apparaît à Drogo son propre destin». «E dal Nord, dal settentrione invisibile dietro le mura Drogo sentiva premere il proprio destino» ${ }^{13}$.

Aldo et Drogo acceptent la vie de garnison à la forteresse et à l'Amirauté même s'il est difficile pour eux de justifier l'existence de ces lieux et surtout le rôle qu'ils y jouent. Dès leur arrivée, ils ouvrent toute grande «la fenêtre de leur pauvre chambre» et parient sur «l'imagination de la nuit». En même temps ils sont en quelque sorte des guetteurs jour et nuit.

Le critique insiste aussi sur l'importance du thème du guet «par quoi s'apparient profondément les deux œuvres. Aux portes du néant sans cesse questionné le veilleur se tient prêt pour la visitation» ${ }^{14}$.

À la «liturgie de l'attente» succède le moment de «l'alerte»; les signes en sont, dans Le Rivages des Syrtes «un navire émergé de la mer» et dans Le Désert des Tartares un cheval pour ainsi dire «né des sables»: convergence apparente mais différence au niveau du message. Tandis que le bateau qui rôde la nuit au large des Syrtes est aux ordres de Vanessa - qui, justement, a un rôle essentiel dans l'expédition navale d'Aldo - le cheval rejeté dans le désert est une «pure épave» ${ }^{15}$.

Aux signaux inquiétants, Aldo, protagoniste actif, réagit en transgressant la «ligne fatale», sorte de frontière maritime tacitement admise par les deux parties, Orsenna et le Farghestan. Il y a bien chez Buzzati «un mouvement: on passe d'un enfermement claustral» à une «immersion dans la vastitude du désert» mais son sens n'est pas celui de Gracq. Sur la frontière Tartare, Drogo, passif, - en fait, il ne sera pour rien dans le déclenchement de l'événement - «découvre une essentielle solitude». Tandis que le fort Bastiani sombre dans la léthargie et la décrépitude, l'Amirauté se réveille, se renforce et Orsenna se met «à guetter, à fixer sur la mer le point d'où jaillira "l'événement", à désirer la fin pourvu que la fin soit un commencement» ${ }^{16}$. Gracq «ménage» une lente mais inéluctable ascension ver la conflagration générale. $\grave{A}$ «la courbe régulière» du roman de Gracq correspond chez Buzzati une ligne brisée avec «chutes... et immobilisation de l'engrenage»; «un abîme de quinze années, creusé en quinze lignes» ${ }^{17}$.

Si le Désert des Tartares apparaît comme une «fable philosophique» ${ }^{18}$ illustrant un destin personnel, traitant un problème «d'enracinement personnel», «Le rivage des Syrtes est aussi un "roman politique" qui engage à travers Aldo un État et un peuple dans leur existence même».

(11) Ibid., p. 92.

(12) Ibid., pp. $92-93$.

(13) D. Buzzati, Il Deserto dei Tartari, Milano, Oscar Mondadori, 1966, p. 68.

(14) «L'Herne», cit., p. 93.
(15) Ibid., p. 95.

(16) «L'Herne», cit., p. 97.

(17) Ibid., p. 99.

(18) Ibid., p. 102. 
En conclusion, l'auteur souligne à la fois la proximité et la différence des deux romans, Gracq pariant sur la «fécondité» et Buzzati sur «l'impuissance du désir». Ce qui réunit néanmoins les deux œuvres est leur «caractère poétique», «non pas dans le style qui n'a rien en commun, mais dans cette attitude générale devant le donné».

Cette attitude trouverait en effet son expression dans le thème du guetteur: «guetteurs tendus vers le tremblement secret des êtres»-selon la belle formulation de Philippe Berthier.

L'association Gracq-Buzzati est encore illustrée par la double participation de Mme Simone Vierne, citée au Colloque International d'Angers sur Gracq (1981) et au Colloque de Grenoble sur Buzzati quelques années plus tard (1986) avec sa communication: «Mort et initiation dans le Désert des Tartares ou le final ambigu» ${ }^{19}$, le roman de Gracq: le Rivage des Syrtes servant en sorte de contrepoint, précise-t-elle. Elle ne s'interdit pas quelques incursions dans un troisième roman, Sur les falaises de marbre d'Ernst Junger et montre le rapport entre les trois romans, en particulier la «quête initiatique» qui les sous-tend.

«Toute quête initiatique tente d'atteindre un Graal qui serait révélation d'une autre manière d'envisager la vie et la mort dans le destin de l'homme».

L'auteur analyse ainsi «le décor et les images» qui illustrent la quête initiatique; et d'abord le roman de Buzzati «plus proche du conte symbolique (jusqu'au chapitre $\mathrm{XXI}$ ), que de la forme romanesque traditionnelle» ${ }^{20}$. La présence du désert, le nom même de l'ennemi nous plonge dès le début dans une «fantastique transcendantale» pour reprendre le terme de Gilbert Durand ${ }^{21}$, c'est-à-dire un univers où le décor naturel, héros, événements, sont avant tout symboliques. «Le désert est le lieu idéal d'initiation où inscrire l'espoir» ${ }^{22}$.

Le décor du Rivages des Syrtes est bien «plus complexe»: «désert et mer», la proximité de Maremma, le lieu du pourrissement qui est fermentation, la forteresse de l'Amirauté, «puissante et complexe».

Ces décors symboliques sont là «pour que s'y passe l'événement» qui changera «le sens même du destin banal en permettant d'échapper au temps». Mais la «quête initiatique s'infléchit dans le livre de Buzzati en s'engageant sur une voie qui la remet en cause, dénégation magistralement orchestrée dans les quatre derniers chapitres $\gg^{23}$. Si tous les événements se précipitent à la fin du Rivages des Syrtes, ceux du Désert des Tartares sont décevants: le cheval, le groupe de Tartares qui n'arrive que pour tracer la frontière. Drogo ne fait que «subir» au Fort Bastiani «englué par la routine»; s'il gagne en grade, il perd en élan, c'est la «mort de ses désirs» ${ }^{24}$. D'ailleurs pour les officiers qui l'entourent, seul Angustina peut devenir un héros en choisissant «le sacrifice volontaire» de sa vie. Et au moment où l'armée du Nord arrivera, Drogo, victime d'une «usure générale», due à «la maladie du temps qui passe», s'évanouit; c'est le contraire de l'évanouissement «initiatique» nécessaire à la «renaissance». A travers les dernières pages du Désert des Tartares, c'est le «dessèchement», l'engourdissement; c'est la fin de Drogo. «C'est une scène de crépuscule» ${ }^{25}$. D'ailleurs au sujet

(19) Simone Vierne, «Cahiers Buzzati» n. 7 (pages 154 à 166), Association des Amis de Dino Buzzati, Paris, 1988.

(20) Ibid., p. 153 et p. 156.

(21) GILBERT Durand, Figures mythiques et visages de l'œuvre, Paris, Berg, 1982. Du même auteur, Les structures anthropologiques de l'imaginaire, $\mathrm{Pa}$ ris, Bordas, 1969.

(22) Simone Vierne, «Cahiers Buzzati», cit., pa- ges 157 à 159 .

(23) Ibid., p. 156.

(24) Ibid., pp. 163 et 159. «Précédé de ce terme essentiel, engluer, le mot habitude précède la description des bruits, des gestes, des rapports sociaux. Elle les souligne et les annule. Une étude des termes de ce champ sémantique serait à mener».

(25) Ibid., p. 164. 
de la «fin» des romans, Mme Vierne précise qu'elle préfère parler de «finale» (au sens musical du terme) «plutôt que de dénouement car, dans les trois romans, rien ne se dénoue au sens strict du terme et particulièrement dans le Désert des Tartares» ${ }^{26}$.

Mais l'attente chez Gracq est grosse d'un nouveau monde et d'un nouvel être; elle révèle la croyance à une «renaissance», non seulement pour les héros mais pour leurs patries et l'auteur joue sur «un registre épique» éloigné du tragique de Buzzati.

Etant donné que les critiques ne s'accordent pas sur le «finale» du roman de Buzzati - sur le sens à lui donner - l'auteur, excluant «une solution univoque à l'arrivée des Tartares et à la mort de Drogo», maintient «l'ambiguïté («finale ambigu») qui d'ailleurs donne sa «poétique grandeur» à ce finale «et nous laisse le choix: vide ou bien Enfer et Ciel?». «Le sourire de Drogo est finalement un sourire de défi: la grandeur du protagoniste vient du renoncement à l'espoir et à l'acceptation du désespoir surmonté» ${ }^{27}$.

Simone Vierne ne s'était pas interdit «quelques incursions» dans un troisième roman, celui de Junger. Patrick Marot annonce comme une nouveauté une «convocation triangulaire» des trois œuvres dans sa communication au Colloque d'Angers sur Gracq en 1992: Figures de la lecture dans trois romans des années de guerre: Sur les falaises de marbre d'Ernst Junger (1939), Le Désert des Tartares de Dino Buzzati et Le Rivage des Syrtes de Julien Gracq.

Il n'est pas étonnant, dit-il, que les «fables des trois récits dont les parutions encadrent le second conflit mondial soient centrées sur l'attente de la guerre». Mais une «mise en œuvre symbolique» instaure une distance par rapport à l'actualité de l'événement «et permet dans les moments les plus tragiques de l'histoire, une lecture symbolique et une visibilité des signes» («une poétique du questionnement») ${ }^{28}$.

A travers les titres, l'auteur cerne l'intérêt du rapprochement de ces romans: le Désert des Tartares et le Rivage des Syrtes dans lesquels «le parallélisme syntagmatique est flagrant»: mais il y a un «espace vide et un en-face», c'est-à-dire une contradiction entre l'annulation de tout événement («la fin de l'espace social») et l'altérité qui permettrait à l'événement de surgir. Si l'altérité apparaît dans le titre de Buzzati, les «Tartares», elle est seulement supposée dans le Rivage des Syrtes à partir du mot «Rivage», le complément de nom («des Syrtes») ne renvoyant qu'à lui-même. Le rapprochement entre les deux récits révèle donc une «problématique de la frontalité: la guerre est dispensatrice de valeurs; régénérer par le feu une civilisation épuisée» (Gracq); «donner un sens à une destinée individuelle menacée par l'insignifiance du social» (Buzzati) ${ }^{29}$. La frontalité de l'espace conditionne l'émergence d'une «altérité héroïque», une rupture du mode de socialité ou d'existence historique présent. Si l'événement est la guerre, il peut être approché, il peut y avoir des changements de signe, comme le dit Vanessa dans le Rivage des Syrtes: «ce qui m'a frappée... c'est qu'il doit y avoir un changement de signe. Un moment où on s'accroche encore, et un moment où on saute» ${ }^{30}$. Des changements anodins dans le quotidien peuvent être des signes redoutés.

Au contraire, dans le Désert des Tartares, les signes ne sont pas indices d'un «bouleversement historique», il s'agit plutôt de «pseudo événements décevants qui finissent par neutraliser l'acte guerrier» ${ }^{31}$. L'objet du désir dans les trois romans est «la

(26) Ibid., pp. 155 et 165.

(27) SimOnE VIERNE, «Cahiers Buzzati», cit., pp. 155 et 166 .

(28) Actes du Colloque International d'Angers sur Gracq (1992), pages 543 à 557, opus cité pp. 543 et 544 .
(29) Ibid., page 545.

(30) J. GracQ, Le Rivage des Syrtes, José Corti, Paris, p. 244.

(31) Actes du Colloque Internationale d'Angers sur Gracq (1992), p. 546. 
plénitude d'une révélation et d'un accomplissement». Dans le Rivage des Syrtes, Aldo est certain du caractère «inéluctable de la guerre»: «un pas à la fin comblait l'attente de cette nuit vide, et je savais pourquoi désormais le décor était planté» et c'est là le finale du roman.

Analysant le problème du temps dans les deux romans, Patrick Marot se distingue d'autres critiques, précisément sur l'importance du temps. Pour le Désert des Tartares, il ne pense pas que le sujet du livre soit «le temps qui passe»; le passé n'existe pas comme tel pour Drogo sinon «pour confronter deux présents distants dans le temps». Il fonctionne alors en dehors de la durée; d'où «le constat d'une universelle déprise temporelle» ${ }^{32}$. Dans le Rivage des Syrtes, le temps est immobilisé par l'affaiblissement des «signes» dû à l'action des «poètes de l'événement» (Aldo, Vanessa, Danielo) qui donnent aux «signes» la dimension d'un horizon et d'un avenir. Ces signes par ailleurs - et dans les deux œuvres - sont réservés aux seuls initiés: en cachette Drogo et Simeoni usent de la longue vue de ce dernier, le règlement du fort n'utilisant que des modèles périmés. Dans le Rivage des Syrtes, les lieux où Aldo peut percevoir les «indices et une altération» sont voués à la solitude et au secret: la chambre des cartes, le chemin de ronde déserté, les ruines de Sagra ou l'île de Vezzano.

Dans ces romans, le récit est la «fable même d'une quête et d'une conquête du sens» assurant une continuité entre l'affirmation symbolique et une progression événementielle largement chronologique ${ }^{33}$. Mais à l'impression d'étouffement chez Buzzati: «on tourne la page, des mois et des années passent» (chapitre XXVII), s'oppose celle d'ouverture croissante et de respiration chez Gracq. Au choix du virtuel chez Buzzati: «l'arrivée des Tartares» s'oppose chez Gracq l'affirmation de la valeur comme résultante d'une «autofécondation poétique» (sens et désignation). «Le Désert des Tartares», note enfin Patrick Marot, «délimite un lieu de problématisation littéraire qui se révèle assez proche de celui du Rivage des Syrtes» ${ }^{34}$.

À propos de la réserve exprimée dans l'analyse précédente sur le «temps comme sujet du livre le Désert des Tartares», il est également intéressant de connaître le point de vue d'Eric Faye, écrivain et essayiste, dans son livre Le Sanatorium des malades du temps s. $^{35}$, Temps, attente et fiction autour de Julien Gracq, Dino Buzzati, Thomas Mann, Kobô Abé» écrit en 1995 et publié en 1996, c'est-à-dire trois ou quatre ans après la communication de Patrick Marot.

Il s'agit bien d'une convocation quadrangulaire à travers cinq romans: Un Balcon en forêt, Le Rivage des Syrtes (Julien Gracq), Le Désert des Tartares (Dino Buzzati), La Femme des Sables (Kobô Abé), La Montagne Magique (Thomas Mann). Nous nous limiterons à l'étude comparative du Rivage des Syrtes et du Désert des Tartares.

Dans son introduction, l'auteur précise: «c'est en pensant à ces sanatoriums pour malades d'un temps spécifique, le temps des machines et de l'intelligence artificielle, qu'ont été rédigées les pages qui suivent». Par ailleurs, si Patrick Marot a choisi «trois romans des années de guerre», ce contexte n'est pas non plus étranger au choix d'Eric Faye: «nous avons choisi de situer autour de la Première Guerre Mondiale la fracture intervenue dans la conception du temps, la date pivot pouvant être cette année 1905 au cours de laquelle Einstein ébauche «la relativité restreinte». Le

(32) Ibid., p. 548, «c'est-à-dire un sentiment ponctuel de paramnésie».

(33) Actes du Colloque International d'Angers sur Gracq (1992), pp. 550 et 551.

(34) Ibid., pp. 555 et 556.

(35) ERIC FAye, Le Sanatorium des malades du temps, Paris, José Corti, 1996.
Il est également l'auteur du roman Le Général Solitude, Paris, Le serpent à plumes, 1995; il a récemment publié Quelques nouvelles de l'bomme, Paris, José Corti, 2009, «dans la veine de Gracq et de Buzzati» (A. DE LARMinat, «Le Figaro Littéraire», 22 octobre 2009 ). 
premier conflit mondial est l'ébauche du second» ${ }^{36}$. La nouvelle époque «de l'atome» règle son divorce avec l'idée de survie après la mort et du paradis. Ce qui apparaissait comme le moment d'un recommencement, la mort, serait donc bien une fin? En précisant la chronologie des cinq romans: La Montagne magique (1924), Le Désert des Tartares (1940), Le Rivage des Syrtes (1951), Un Balcon en forêt (1958) et La Femme des sables (1964), il désigne ceux-là comme «les fruits amers d'une telle époque, sans précédent» ${ }^{37}$.

Sur «une toile de fond commune» aux romans étudiés, l'auteur note une lente montée des périls avec Buzzati comme avec Gracq, une «poussée de fièvre» selon le titre du sixième chapitre du Rivage des Syrtes: «quelque chose comme une alerte lointaine se glisse jusqu’à nous dans ce vide clair du matin plus rempli de présages que les songes» ${ }^{38}$. Le terme de «présages» semble bien choisi pour la «montée en puissance chez Buzzati de la menace» tant est riche le champ lexical de l'angoisse dans Le Désert des Tartares. Mais Eric Faye prend soin de préciser que le roman de Buzzati n'est en rien «une métaphore de montée des périls: il tire profit de ce climat à la seule fin d'aider Buzzati à mettre en place une problématique de l'homme et du temps» ${ }^{39}$.

Néanmoins il ne faut pas oublier que le Désert a été publié sur la «toile de fond» du fascisme: la question d'un anti-fascisme «sous-jacent» a divisé les critiques. Plusieurs ont nié toute intention de ce type. D'autres comme Yves Panafieu «font une lecture politique poussée et s'ils ne privilégient pas, en fin de compte, l'aspect métaphysique du roman, leurs arguments tendraient à faire du roman une bible antifasciste» ${ }^{40}$.

Mais la démonstration de Panafieu est «éloquente» lorsqu'il développe l'idée du «regard impossible» depuis le Fort Bastiani en pleine brume parce que «peu à peu les longues-vues de qualité sont confisquées, parce que l'unique fenêtre donnant sur la plaine se trouve dans le bureau du Colonel (comprendre du Duce, celui qui voit loin et conduit) tandis que la lucarne de la chambre de Drogo donne sur la cour intérieure».

Pourquoi alors ce roman est-il situé en milieu militaire? Buzzati y répond luimême: «je pensais que dans un milieu militaire, mon histoire aurait pu aller jusqu'à acquérir la force d'une allégorie concernant tous les hommes» ${ }^{41}$. Et Eric Faye conclut cette «parenthèse sur le fascisme» en pensant que le Désert porte en filigrane une condamnation de la rhétorique de «l'héroïsme belliqueux», selon l'expression d'Yves Panafieu ${ }^{42}$.

Revenant à son discours sur le temps, l'essayiste nous conduit au tragique car «les dieux hostiles ou le fatum ont pris l'aspect simple et terrible du temps». Ce qui est attendu dans ces romans, «c'est le pire, c'est la mort». Cette attente permet une mise sous tension du récit, chère à l'art de Gracq. Le combat oppose désormais «l'humain à quelque chose de supra-divin et d'indéfinissable: le temps»; «la propension à renouer avec la veine tragique est grande chez Gracq et chez Buzzati» qui ont construit une œuvre «à l'écart» de la production de leur époque ${ }^{43}$.

Le temps est précisément au cœur de ce qui distingue les deux romans, le Rivage et le Désert. Si dans le Désert, «le cours du récit est ponctué de brefs développements

(36) E. FAYE, op. cit., pp. 12 et 19.

(37) Ibid., p. 19.

(38) Le Rivage des Syrtes, p. 109.

(39) E. FAYE, op. cit., p. 75.

(40) Ibid., page 76. Pour information et par nos soins, voir le «Magazine Littéraire», octobre 1995: Yves Panafieu, Une lecture socio-bistorique du "Désert des Tartares", pp. 42-43.
(41) M. Suffran, Dino Buzzati, Entretien avec Yves Panafieu, Lyon, La manufacture, 1988, coll. «Qui êtes-vous?» n. 34, p. 247.

(42) E. FAYE, op. cit., page 77. Voir «Le Désert des Tartares, un temps et un espace de crise» d'Yves Panafieu, Colloque de Milan 1982.

(43) E. FAYE, ibid., pp. 90, 91 et 105. 
sur le temps», dans le Rivage, ces digressions n'ont pas lieu d'être. Si dans le Rivage le temps est envisagé dans une «perspective historique», dans le Désert, il n'a de sens qu'étudié au niveau de l'individu. Toutefois, entre Aldo et Drogo, il y a une passion commune: «celle-ci réside peut-être dans ce pouvoir de rétention de l'extraordinaire: voir avant mais ne pas avertir les autres: savoir seul» ${ }^{44}$.

S'il y a des «sautes de temps» dans les deux romans, six à sept siècles pour les guerriers «tartares - mongols», trois siècles pour le conflit avec le Farghestan, la datation est, de toute façon, inutile car l'on n'est pas dans le temps propre à l'histoire chronologique de l'humanité mais bien ailleurs, dans un «présent éternel propre aux mythes», «dans le combat de la civilisation face aux Barbares»; le mythe est là, même dissimulé derrière des forêts de dates; ces romans reposent sur une superposition du «temps de l'éphémère» à celui de l'individu, qui chute dans l'abîme de la mort et du «présent éternel», ce dernier faisant mieux apparaître le premier.

Il n'est plus question de nostalgie, la fuite du temps s'exprime davantage dans la prise de conscience du temps qui reste, «durée vague qui peut aller de l'éphémère à de longues années» ${ }^{45}$. Ce vers quoi les héros de ces romans «regardent avec angoisse, c'est la ligne d'horizon, non pas celle qu'ils ont dans le dos, mais devant eux, celle de l'avenir, synonyme de mort» ${ }^{46}$. Mais pour voir cette ligne d'horizon, vivre le «temps qu'il reste», ils ont besoin d'être seuls en face d'un désert, par exemple «lieu où on ne va pas, donc par définition le lieu de l'imaginaire et du magique». C'est pourtant de là que viendra l'ennemi et la mort. «Le désert est la matrice des peuples envahisseurs» ${ }^{47}$.

Eric Faye intègre ici une partie de l'analyse d'Yves Frontenac, déjà cité dans notre introduction à propos des sources et des influences. Frontenac explique que le Désert des Tartares, le Rivage des Syrtes sont des «romans du vide» et cite à ce sujet une phrase du Désert: «la forteresse apparaissait comme un mur très long. Un simple mur, avec, à l'intérieur, rien» ${ }^{48}$.

Enfin, dans le chapitre de conclusion de son essai, Eric Faye pose la question à propos de «la ligne d'horizon» évoquée précédemment. «Un Dieu (ou quelque absolu) est-il vraiment cantonné derrière l'horizon que scrutent Aldo, Drogo?» et poursuit «les héros - victimes de ces romans sur le temps ont souffert, ont attendu et souffert d'attendre. La plupart d'entre eux, en dernière instance, vont guérir. Ils ont pénétré depuis longtemps les sanatoriums pour malades du temps. Le traitement porte ses fruits. La délivrance... Mais la convalescence, si tant est que les soins aient suffi, ne débute guère qu'au moment où le patient est en vue de la mort» ${ }^{49}$.

Après la «graduation du Désert» d'Éric Faye, nous allons précisément achever notre présentation sur le «Désert». Dans son article, «Écrire le désert: Buzzati, Gracq, Camus», Mme Delphine Gachet précise d'emblée ce qu'elle entend par «écrire le désert»: «mettre l'espace désertique au cœur de la fiction narrative et assumer les conséquences littéraires de ce choix, c'est-à-dire un certain type de littérature». Il s'agit d'éclairer l'œuvre de Buzzati (Le Désert des Tartares) en la confrontant à deux de ses contemporains français: Gracq dans le Rivage des Syrtes et Camus dans l'Exil et le Royaume $e^{50}$.

(44) Ibid., pp. 130 et 133.

(45) Ibid., pp. 139,140 et 211.

(46) Ibid., p. 212.

(47) Ibid., p. 171. Pierre Jourde, Géographies imaginaires de quelques inventeurs de mondes au $\mathrm{XX}^{\mathrm{e}}$ siècle, Paris, José Corti, 1991, p. 52.

(48) E. FAYE, op. cit., page 175. Eric Faye consa- cre dans son essai un chapitre au Désert: «La graduation du désert», pp. 143-150. Voir aussi YVES Frontenac, Sources du "Désert des tartares" et du "Rivage des Syrtes", «Cahiers Dino Buzzati» n. 5 (1982), pp. 49-60.

(49) E. FAYE, op. cit., page 222.

(50) D. GACHET (secrétaire de l'Association In- 
Comment est-on conduit à l'écriture du désert? En partant de l'expérience du désert, c'est-à-dire d'un «sentiment», pour en arriver à l'imagination du désert. Ainsi si le désert buzzatien tire son origine de la double expérience italienne (le Piave) et africaine (voyages - journalisme du guerre), le paysage du Rivage des Syrtes tire lui aussi son origine de «données empiriques»: «les étendues marécageuses de la campagne belge, le climat brumeux et humide». L'écriture du roman sera donc «tendue par cette volonté de faire revivre l'image première et le sentiment de celle-ci» ${ }^{51}$.

Mais écrire le désert ne relève pas seulement de l'effort autobiographique, c'est aussi «créer un espace à mi-chemin entre le réel et le fantastique». Si le désert est un espace vide, «la vacuité doit être lue comme métaphore de l'apparente absence du sens». Stimulation à la découverte, mer et désert de sable ou de pierre dont la correspondance dans la structuration de l'imaginaire est soulignée, offrent un terrain fertile à la croissance des rêves et des angoisses, «royaumes des désirs et des peurs pour les écrivains». Métaphores et images font du désert un «espace cosmique». On retrouve aussi bien dans le Désert que dans le Rivage l'image de «l'immensité du ciel nocturne avec les myriades d'étoiles des désirs humains ${ }^{52}$.

Dans la recréation du désert opérée par le passage à la fiction, l'écrivain va détacher le désert de son ancrage dans le réel. Buzzati et Gracq «subvertissent l'inscription réaliste de leur roman». Les titres semblent accorder la primauté à l'espace: «désert», «rivage» mais le paysage décrit ne semble pas réel. Ainsi le titre Le Désert des Tartares n'a pas de réelle référence géographique mais l'espace mythique d'un peuple, les Tartares. Dans le Rivages des Syrtes aussi le paysage est différent de sa référence méditerranéenne. Le pays qui fait face, comme chez Buzzati, est le lieu d'où vient la menace. L'auteur de l'article souligne ce double mouvement d'inscription et de distanciation par rapport à l'espace réel, «le désert étant à la fois nulle part et partout» ${ }^{53}$.

Écrire le désert est aussi un choix d'ordre littéraire: la nécessaire adéquation entre les lieux du récit et le récit lui-même. «La vacuité est au centre de la problématique». Quelles seront les caractéristiques du récit? Le dépouillement de «l'intrigue des actions et des péripéties». Il y a certes une différence entre le roman de Gracq avec une abondance d'actions, de personnages, de lieux et celui de Buzzati plus sobre mais l'intrigue chez Gracq est également réduite. «S'il y a une relation entre la vacuité de l'espace et l'amenuisement de l'intrigue, la réduction de l'effectif romanesque dans le récit doit aussi en être la conséquence; les romans du désert proposent des figures du solitaire et c'est à travers les yeux du personnage principal que le monde est perçu» ${ }^{54}$. Ce personnage est isolé par rapport aux autres qui sont parfois des projections du personnage principal: Fabrizio dans le Rivage et Angustina dans le Désert. La solitude des protagonistes est renforcée par ceux qui s'opposent à eux, «il Maggiore Mattiera era grassoccio, il suo ufficio era vasto, grande era pure la scrivania, ingombra ordinatamente di carte», et pour qui le désert ne représente rien: «un paesaggio monotono, non c'è proprio niente di bello» ${ }^{55}$. D'ailleurs c'est précisément parce qu'ils ont face au désert une attitude différente que les personnages principaux sont isolés. Seuls ceux qui, aux portes du désert, «regardent le désert vont pouvoir lire le désert». Ainsi les récits du désert sont des récits de la quête. Buzzati n'avait-il pas pensé à un titre pour son roman «Le Message du Nord» ${ }^{56}$ - de la quête du signe.

ternationale des Amis de Buzzati, doctorat littérature comparée, Fantastique italien et français au XX siècle, Université de Bordeaux), «Narrativa» n. 23, CRIX, Paris X Nanterre, mai 2002, pp. 99-104.

(51) Ibid., p. 3.

(52) Ibid., p. 4.
(53) Ibid., pp. 4 et 5 .

(54) Ibid., p. 6.

(55) Il deserto dei Tartari, cit., pp. 21 et 26.

(56) Article page 8. Voir aussi M.H. CASPAR, Fantastique et mythe personnel dans l'auvre de Buzzati, $\mathrm{La}$ Garenne-Colombe, Editions de l'espace euro- 
Drogo, à la forteresse, constate l'absence de sens mais a cet «obscur pressentiment des choses fatales». Aldo, arrivant aux Syrtes, éprouve un sentiment analogue: «quelque chose m'était promis, quelque chose m'était dévoilé... j'attendais le matin... comme on s'avance les yeux bandés vers le lieu de la révélation» ${ }^{57}$. Mettre du sens là où il n'y en a pas, trouver «les signes graphiques ou sonores d'un message qui échappe aux autres». Ainsi dans le Désert des Tartares les images du «nuage blanc» et de la «tache», du «point» ou de la «ligne noire» ${ }^{58}$.

Si l'écriture du désert conditionne «un certain type de littérature» annoncé dans l'introduction de cet article, après la confrontation de Buzzati avec Gracq et Camus, la singularité en est soulignée dans la conclusion: «le caractère hétérogène de l'espace mis en scène et l'importance de la description». Vacuité du désert et mise en scène de l'espace suscitent «chez le personnage de l'homme du désert un flottement du sens et des sens, faisant du récit du désert avant tout celui d'un regard. Ce regard, fondement de l'écriture du désert, peut aller jusqu'à faire du personnage un «guetteur-sémiologue» ${ }^{59}$, selon l'heureuse expression de Madame Gachet.

Les auteurs des communications précédentes ne manquent pas de mentionner les articles les plus éloignés dans le temps et totalement consacrés à la comparaison: «Le Rivage des Syrtes et le Désert des Tartares», comme celui de Ph. Berthier (1972) et plus tard ceux de Yves Frontenac et Yves Panafieu (entre 1980, 1995 jusqu'à nos jours). On peut certes constater un moindre intérêt porté à la seule confrontation des deux œuvres et une évolution vers «une convocation triangulaire» selon l'expression de P. Marot, amorcée déjà par Mme Simone Vierne (1981 - 1986) avec son allusion à Junger (Sur les falaises de marbre) et annoncée et développée plus nettement avec force d'arguments par P. Marot en 1992. Mme Gachet plus récemment (2002) se situe dans cette voie avec toutefois un éclairage particulier car il s'agit, dit-elle, «d'éclairer l'œuvre de Buzzati». Le mouvement d'élargissement se poursuit avec une «convocation quadrangulaire» d'Eric Faye dans son essai: quatre œuvres citées (dont une allemande et une japonaise), avec une place assez large accordée aux deux romans de Gracq et de Buzzati.

Néanmoins pendant cette période on trouve encore des travaux consacrés à ces derniers en $1988^{60}$ et en 1997 un mémoire de maîtrise ${ }^{61}$ non dénué d'intérêt. Certes il ne s'agit pas d'un catalogue des ressemblances et des différences mais d'un approfondissement, d'une pluralité de significations.

Des divergences apparaissent sur l'importance du temps, sur le contenu du message - selon les années - politique ou individuel, sur l'interprétation de la «quête», le comportement des protagonistes en face de la mort, le caractère «poétique» des deux œuvres malgré la différence totale des deux écritures semble être reconnu par tous les critiques et «l'élargissement» favorable aussi à la fécondité de l'imagination ne nuit pas à l'affinement de l'analyse.

NORBERT GERMANAZ

péen, Erasme 1990, page 183: «Resémentisation du désert».

(57) Le Rivage des Syrtes, cit., p. 18.

(58) D. GACHET, cit., page 9.

(59) Ibid., p. 10.

(60) Lorella SinI, La quête du sens dans "Le Ri- vage des Syrtes" de Julien Gracq et le "Désert des Tartares" de Dino Buzzati (Université de Nice, 1988).

(61) Le désir d'aventure dans le Désert des Tartares et Le Rivage des Syrtes présenté par Arnaud GADY (Littérature Comparée, Université de Limoges, 1997). 